\title{
Manejo anestésico en el paciente pediátrico con COVID-19
}

\section{Anesthetic management in the pediatric patient with COVID-19}

Dr. Gabriel Mancera-Elías,* Dra. Alma Dolores Arenas-Venegas ${ }^{\ddagger}$

RESUMEN. La infección por virus SARS-CoV-2 tiene un comportamiento diferente en pacientes pediátricos, por lo que el abordaje anestésico puede diferir del que se realiza en adultos. El presente trabajo toca algunas generalidades del manejo anestésico del paciente pediátrico que deben ser consideradas.

ABSTRACT. Infection with SARS-CoV-2 virus has a different behavior in pediatric patients, so the anesthetic approach may differ from that performed in adults. The present work touches on some generalities of anesthetic management of the pediatric patient that should be considered.

\section{GENERALIDADES}

$\mathrm{E}$ l virus SARS-CoV-2 aislado en un paciente en Wuhan, China, es un nuevo coronavirus ARN beta con un elevado potencial de infección y que ha sido denominado síndrome respiratorio agudo por coronavirus (SARS-CoV-2) o enfermedad por coronavirus (COVID-19). El 30 de enero de 2020 la Organización Mundial de la Salud anunció al brote de COVID-19 una emergencia pública y en marzo alcanzó el grado de pandemia.

Hay dos situaciones relacionadas a su modo de transmisión que son de particular interés para el anestesiólogo. La exposición a gotas (usualmente pueden dispersarse en un radio de $2 \mathrm{~m}$ cuando tosen o estornudan los pacientes infectados) y la generación de aerosoles (cuyas pequeñas partículas pueden penetrar las mascarillas estándar y tener un mayor radio de dispersión) $)^{(1)}$; los procedimientos que generan aerosoles pueden incluir a la intubación endotraqueal, la ventilación no invasiva, el alto flujo nasal, broncoscopías, succión bronquial, inducción de esputo, entre $\operatorname{otros}^{(1)}$. En la Tabla 1 se observan más causas de procedimientos asociados a aerosolización ${ }^{(2)}$.

Las manifestaciones clínicas son variables que pueden atribuirse a diferencias estructurales a las proteínas virales afectando su tropismo y replica ${ }^{(3)}$. Es de especial preocupación para el grupo de anestesiólogos el hecho de que algunos pacientes pueden presentar sintomatología mínima o abdominal ${ }^{(3)}$.

El cuadro clínico su puede observar en la Tabla $1^{(4,5)}$.

El cuadro clínico puede diferir al de los adultos quienes presentan mayor incidencia de tos, fiebre y dificultad respiratoria y en casos severos presentan disnea pudiendo progresar a síndrome de insuficiencia respiratoria del adulto y otras complicaciones como inflamación sistémica y choque séptico ${ }^{(5)}$. En el paciente pediátrico los cuadros respiratorios pueden durar de una a dos semanas, pocos desarrollan insuficiencia respiratoria y pueden incluir casos especiales como lactantes, pacientes con patología respiratoria previa, cardiopatía o pacientes inmunocomprometidos ${ }^{(5)}$.

Ho y colaboradores ${ }^{(6)}$ refieren que dentro de las posibles causas de las variaciones en los cuadros clínicos entre la presentación entre pacientes pediátricos y adultos entre ellas la reducción en la exposición de niños básicamente a su comunidad, otras explicaciones incluyen el poseer una inmunidad inmadura a las infecciones virales y por consecuencia responder de manera diferente a los adultos. Estudios han concluido que el coronavirus (SARS-CoV) y el nuevo coronavirus
Anestesiología

Octubre-Diciembre 2020

Vol. 43. No. 4. pp 325-328

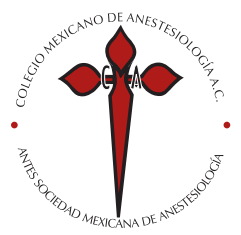

Palabras clave:

Infección SARS-CoV-2,

COVID-19, anestesia,

paciente pediátrico.

Keywords:

SARS-CoV-2 infection,

COVID-19, anesthesia, pediatric patient.

\footnotetext{
* Médico de Base Departamento de Anestesiología. Departamento de Anestesiología. Instituto Nacional de Pediatría S.S.

${ }^{\ddagger}$ Médico de Base Departamento de Anestesiología. Unidad de Pediatría. Hospital General de México, S.S.
}

Solicitud de sobretiros: Dr. Gabriel Mancera Elías Instituto Nacional de Pediatría. Insurgentes sur 3700 Letra C, Insurgentes Cuicuilco, Ciudad de México. C.P. 04530

E-mail:

elimanga81medicina@gmail.com

Recibido para publicación: 14-07-2020

Aceptado para publicación: 26-07-2020 
(2019-nCoV) usan el mismo receptor enzima convertidora de angiotensina II (ACE-II), la cual es posible que en niños no posea los mismos estándares que en los adultos.

Los conceptos anteriores nos dejan una serie de consideraciones en qué reflexionar: la posibilidad de enfrentar a portadores asintomáticos, la disparidad de síntomas que pueden llevar a confusión relacionada a la positividad del caso, el escenario de cirugía urgente versus urgencia relativa y el sitio donde se llevará a cabo el acto anestésico, quirófanos o procedimiento fuera de (tomografías, resonancias, etcétera), así como la posibilidad de procedimientos endoscópicos. Lamentablemente no se pueden tocar todos los tópicos a profundidad, pero es fundamental establecer que los universos de trabajo son diversos y cada situación involucra diferencias en su manejo ${ }^{(7)}$.

\section{VALORACIÓN PREANESTÉSICA}

La evaluación debe ser integral, abarcando no únicamente lo referente a las manifestaciones propias de COVID-19, deberá incluir las comorbilidades al igual que la patología primaria que requiere la intervención asociada, sea únicamente la respiratoria o adjunta a patología quirúrgica, los exámenes de laboratorio pertinentes y la prueba específica para SARS$\mathrm{CoV}-2$ y/o un estudio radiológico o tomográfico ${ }^{(8,9)}$.

\section{LA TÉCNICA ANESTÉSICA}

Controlar la ansiedad del niño es fundamental, los niños pueden manifestar la ansiedad en el preoperatorio como llanto excesivo y agitación, situaciones que incrementan la generación de aerosoles ${ }^{(10,11)}$; estas situaciones de estrés adicionalmente se acompañan de un incremento en el tiempo de inducción ${ }^{(11)}$. A fin de evitar esta situación la Sociedad Española de Anestesia Reanimación y Terapéutica del dolor (SEDAR) emite una serie de recomendaciones relacionadas a la técnica anestésica y al abordaje de la vía aérea; en el punto referente a la ansiedad la recomendación es la premedicación a través de la vía oral, ya sea mediante clonidina, dexmedetomidona o midazolam ${ }^{(10)}$, la vía nasal no es recomendable debido a que en pacientes con carga viral alta existe el riesgo de tos y estornudo con la consecuente aerosolización ${ }^{(12)}$.

Las recomendaciones generales enfatizan el uso del equipo de protección personal para el equipo involucrado en el manejo anestésico, la sala quirúrgica especialmente designada para los casos COVID-19 debe estar equipada con un equipo de presión negativa, si el quirófano designado no contara con ésta, se deberán apagar los equipos de presión positiva y el aire acondicionado ${ }^{(10,13)}$, la máquina de anestesia debe permanecer con doble o triple HEPA reemplazándolo cada tres o cuatro horas de anestesia ${ }^{(13)}$, asimismo, se debe tener preparado el equipo de abordaje de vía aérea, los elementos de aislamiento del paciente, la preparación de los medicamentos previo a entrar a la sala a fin de evitar caída de líquidos al piso durante el procedimiento para evitar contaminación y todas las medidas preoperatorias pertinentes a cada caso.

A pesar de que la inducción inhalatoria es la técnica más utilizada en la población pediátrica, existe la posibilidad de generación de gotas y aerosoles, por lo que la inducción intravenosa es preferida ${ }^{(12,13)}$, la inducción de secuencia rápida y la secuencia modificada son útiles; sin embargo, existe la posibilidad de una hipoxemia severa en pacientes pequeños, hay que recordar que en neonatos y lactantes existen factores fisiológicos que deben considerarse sumado a las diferencias anatómicas de la vía aérea superior, como un desarrollo de la musculatura intercostal y mayor dependencia del diafragma, costillas horizontalizadas y menor capacidad residual funcional, mayor volumen de cierre y un consumo metabólico de oxígeno más elevado ${ }^{(14)}$. Los pacientes con patología pulmonar también pueden manifestar hipoxemia, de tal modo que estos pacientes recibirán una ventilación suave hasta elevar la jaula torácica mientras se mantiene un sellado de la mascarilla (técnica con dos anestesiólogos) ${ }^{(12)}$.

Tabla 1: Cuadro clínico de COVID-19.

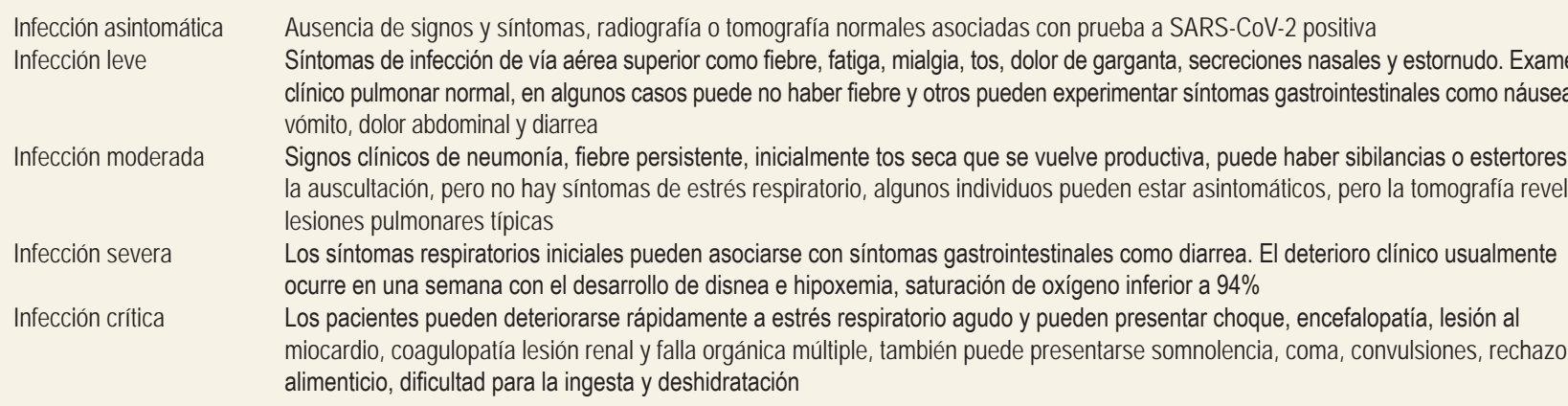

Modificado de: Management Pediatric COVID-19 Carlotti APCP et al. Clinics. 2020; 75: e1894.

Bernucci MF, Fajardo A, Maureira V, Heider RM. Anestesia para pacientes pediátricos con COVID-19. Rev Chil Anest. 2020; 49: 422-432. 
A pesar de esta preferencia, puede existir el escenario del paciente que se presente en la sala de operación sin un acceso venoso teniendo que recurrir a la inducción inhalatoria donde se recomienda el uso de flujos bajos con ventilación espontánea para evitar la ventilación con mascarilla y así prevenir la aerosolización. En el caso de tener que recurrir a la ventilación del paciente, de modo similar a como se encuentra descrito algunos renglones arriba se recurrirá a la técnica con dos anestesiólogos ${ }^{(10,12)}$. El punto importante con el asunto de aerosolización lo describe Hirota ${ }^{(15)}$, donde describe que por su afinidad a la enzima convertidora de angiotensina 2 el virus puede tener una expresión alta en células que contienen el gen receptor de la enzima convertidora de la angiotensina 2 como neumocitos, epitelio bronquial, cavidad nasal, epitelio olfatorio, esófago, músculo liso intestinal, neuronas, hígado y riñones.

La ventilación por mascarilla, la succión y la lucha durante el proceso de intubación y extubación pueden producir aerosoles. Guo referido por Hirota ${ }^{(15)}$ reportan la distribución del virus en el quirófano al colectar muestras detectadas en el aire a cuatro metros de los pacientes con COVID-19(14), de ahí la importancia de tener cuidado al momento de la ventilación, la relajación neuromuscular profunda es recomendable ${ }^{(13)}$ y el uso de barreras antes de la instrumentación como se encuentra descrito en las distintas guías de manejo ${ }^{(10,12)}$.

No existe duda que, en el sentido de los anestésicos inhalados, el sevoflurano es el agente de elección, dado a la elevada pungencia del desflurano que puede propiciar incremento de secreciones, apnea, tos y laringoespasmo; sin embargo, el uso del desflurano es considerado seguro en términos de irritabilidad, únicamente en el mantenimiento de la anestesia en pacientes intubados ${ }^{(16)}$. La mayoría de los estudios apuntan hacia un efecto protector contra la broncoconstricción, aunque incrementa la resistencia de la vía aérea después de la inducción y la intubación, mientras que el desflurano es más controversial, carece de efecto sobre el tono basal o elevado del tono de la vía aérea aunque irrita la vía aérea, lo cual se manifiesta por elevaciones de la resistencia de las vías respiratorias ${ }^{(17,18)}$.

Referente al uso de anestesia endovenosa, Chokshi y colegas, en un trabajo de revisión sobre las ventajas del uso de la anestesia endovenosa en pacientes con COVID-19, recomiendan su uso en combinación con anestesia regional o neuroaxial y destaca la factibilidad de uso, inclusive en áreas remotas fuera de quirófano como hemodinamia, tomografía, resonancia magnética y cateterismo cardíaco, al igual que en procedimientos quirúrgicos ambulatorios y en sitios donde la administración de los agentes inhalados sea difícil ${ }^{(19)}$; destaca la ventaja de minimizar la vaporización, menor efecto sobre el sistema inmune, no riesgo de hipertermia maligna, despertar con menor incidencia de delirio de emergencia comparado con los agentes inhalados con menor riesgo de vaporización, menor riesgo de náusea y vómito postoperatorio.

El manejo ventilatorio transoperatorio recomendado incluye una ventilación con volumen corriente bajo $(4-8 \mathrm{~mL} / \mathrm{kg}$ de acuerdo con las recomendaciones europeas y norteamericanas o menos de $6 \mathrm{~mL} / \mathrm{kg}$ de acuerdo a las recomendaciones inglesas, mantener presiones de meseta por debajo de $30 \mathrm{~cm}$ $\mathrm{H}_{2} \mathrm{O}$ ) con hipercapnia permisiva ${ }^{(13)}$.

Referente a la anestesia regional las sociedades americana y europea de anestesia regional publican las recomendaciones de anestesia neuroaxial y de bloqueos periféricos durante la pandemia ${ }^{(20)}$, de modo similar al planteamiento de la anestesia endovenosa el uso de la anestesia regional puede en algunos escenarios evitar la instrumentación de la vía aérea con la consecuente reducción del riesgo de aerosolización, permitiendo, según el caso, que el paciente pueda mantenerse con ventilación espontánea con el uso de puntas nasales con flujos bajos, los procedimientos deberán realizarse en quirófano con el protocolo correspondiente para pacientes COVID. Cuando se utilice el equipo de ultrasonido incluyendo el transductor debe protegerse de la contaminación utilizando proyectores de plástico, con el uso de anestesia espinal no hay suficiente evidencia al momento que la contraindique y las indicaciones y contraindicaciones serán las usuales del procedimiento, se recomienda reducir la posibilidad de contaminación al no permitir la salida libre del líquido cefalorraquídeo, actualmente no hay contraindicación en el uso de las dosis habituales.

Relacionado al manejo de la cefalea postpunción no hay guía disponible en el manejo de los pacientes con COVID-19, las medidas conservadoras son el manejo inicial. El bloqueo esfenopalatino al ser realizado vía nasal incrementa el riesgo de transmisión de la enfermedad, por lo que debe ser evitado en este grupo de pacientes. Existe preocupación relacionada a la inyección de sangre virémica en el espacio peridural si se necesita un parche hemático, si el caso lo requiriera habrá que valorar el riesgo/beneficio de su uso ${ }^{(20)}$.

En el caso del bloqueo de los nervios periféricos, la sedación previa al bloqueo es recomendada, la técnica es la misma que regularmente se utiliza y las dosis son calculadas a las habituales ${ }^{(20)}$.

Para la extubación es recomendable minimizar los accesos de tos, la agitación postanestésica y la exposición a secreciones y aerosoles, considerándose las medidas físicas de aislamiento, así como el uso de dexmedetomidina, lidocaína u opioides, profilaxis antiemética y aspiración con circuito cerrado de succión para reducir los aerosoles ${ }^{(5)}$.

Los esquemas analgésicos son similares a los habituales ajustando las dosis a fin de evitar depresión respiratoria, náusea o vómito.

La recuperación se realizará en la sala de operaciones hasta su traslado a hospitalización o al término del procedimiento se trasladará a la UCI. 


\section{CONCLUSIÓN}

La infección por COVID-19 ha producido dramáticos cambios en el mundo de la medicina, el paciente que requiere procedimientos anestésicos no es la excepción; si bien, la mayoría de los protocolos van encaminados a las medidas de control de infección y de abordaje de la vía respiratoria, deben ser considerados escenarios fuera de quirófano que requieren de atención. La literatura recomienda que los procedimientos radiológicos se realicen con sedación; sin embargo, habrá ocasiones en los que la vía respiratoria tenga que ser o esté instrumentada y tendrán que tomarse las atenciones pertinentes.

Situaciones como dosificación, manejo hídrico y esquemas de analgesia tienden a ser conservadoras, pero deberán ser individualizadas de acuerdo a las patologías subyacentes.

Finalmente, no podemos pasar por alto que los grupos etarios en el paciente pediátrico son amplios y cada adecuación de material, cuidados, técnicas y dosificación deberá corresponder al grupo de edad de cada paciente.

\section{REFERENCIAS}

1. Odor PM, Neun M, Bampoe S, Clark S, Heaton D, Hoogenboom EM, et al. Anaesthesia and COVID-19: infection control. British Journal of Anaesthesia. 2020;125:16-24.

2. Thomas-Rüddel D, Winning J, Dickmann P, Ouart D, Kortgen A, Janssens U. Coronavirus disease 2019 (COVID-19): update for anesthesiologists and intensivists. Anaesthesist. 2020. https://doi. org/10.1007/s00101-020-00760-3.

3. Greenland JR, Michelow MD, LW, London MJ. COVID-19 infection implications for perioperative and critical care physicians. Anesthesiology. 2020;132:1346-1361.

4. De Carvalho PA, Brunow CW, Johnston C, Souza RI, Figueiredo DA. COVID-19 diagnostic and management protocol for pediatric patients. Clinics. 2020;75:e1894. doi: 10.6061/clinics/2020/e1894.

5. Bernucci M, Fajardo A, Maureira V, Heider RM. Anestesia para pacientes pediátricos con COVID-19. Rev Chil Anest. 2020;49:422-432.

6. Tung HCL, Oligbu OP, Pervaiz M, Oligbu G. Clinical characteristics of children with COVID-19. AIMS Public Health. 2020;7:258-273. doi: 10.3934/publichealth.2020022.

7. Mahmoud AH. Perioperative care of pediatric anesthesia for children with suspected or confirmed COVID-19. Saudi J Anaesth. 2020;14:370-377.

8. Huangc J, Hu Y, Wang J, Wang D, Zhu Z, Hu Z, et al. Recommendation about the perioperative prevention of infection to healthcare workers and the anesthesia management of children with SARS-CoV-2 infection. World Jnl Ped Surgery. 2020;3:e00126. doi: 10.1136.

9. Lee-Archer P, Von Ungern-Sternberg BS. Paediatric anaesthetic implications of COVID-19. A review of current literature. 2020. doi: 10.1111/PAN.13889.

10. Hervias SM, Ferreras VR, Sánchez AA, Prada HG, Gálvez EI, Fernández $\mathrm{JM}$, et al. Documento Consenso sobre las principales recomendaciones en el manejo anestésico del paciente pediátrico en el contexto actual de pandemia por Covid19. Marzo 2020. Disponible en: www.sedar.es.

11. Das S, Kumar A. Preoperative anxiety in pediatric age group- a brief communication. J Anesth Crit Care Open Access. 2017;8:00317.
12. Matava CT, Kovatsis PG, Lee JK, Castro P, Denning S, Yu J, et al. Pediatric airway management in COVID-19 patients: Consensus Guidelines From the Society for Pediatric Anesthesia's Pediatric Difficult Intubation Collaborative and the Canadian Pediatric Anesthesia Society. Anestesia \& Analgesia. 2020;131:61-73.

13. Chen X, Liu Y, Gong Y, Guo X, Zuo M, Li J, et al. Perioperative management of patients infected with the novel Coronavirus recommendation from the joint task force of the Chinese society of anesthesiology and the chinese association of anesthesiologists. Anesthesiology. 2020;132:1307-1316.

14. Saikia D, Mahanta B. Cardiovascular and respiratory physiology in children. Indian J Anaesth. 2019;63:690-697.

15. Hirota K. Air contamination with SARS-CoV-2 in the operating room. Journal of Anesthesis. 2020:1-4. https://doi.org/10.1007/s00540-02002814-7.

16. Eun-Hee Kim, In-Kyung Song, Ji-Hyun Lee, Hee-Soo Kim, HyunChang Kim, Soo-Hyuk Yoon, MDa, et al. Desflurane versus sevoflurane in pediatric anesthesia with a laryngeal mask airway a randomized controlled trial. Medicine (Blatimore). 2017;96:35(e7977). http://dx.doi. org/10.1097/MD.0000000000007977.

17. Von Ungern-Sternberg BS, Saudan S, Petak F, Hantos Z, Habre W. Desflurane but not sevoflurane impairs airway and respiratory tissue mechanics in children with susceptible airways. Anesthesiology. 2008;108:216-224.

18. Rajesh MC. Anaesthesia for children with bronchial asthma and respiratory infections. Indian J Anaesth 2015;59:584-8.

19. Chokshi T, Channabasappa S, Vergheese DC, Singh BS, Gupta B, Mehdiratta L, et al. Re-emergence of TIVA in COVID times. Indian J Anaesth. 2020;64: S125-31.

20. Uppal V, Sondekoppam RV, Lobo CA, Kolli S, Kalagara HKP. Practice recommendations on neuraxial anesthesia and peripheral nerve blocks during the COVID-19 pandemic. COVID-19 Guidance for Regional Anesthesia, 2020. 